\title{
Severe Life-threatening Hypercalcemia in Skeletal Metastasis from Malignancy of Unknown Origin: A Rare Presentation
}

\author{
Rajeev Gupta ${ }^{1}$, Srishti Nayyar², Shveta Mahajan ${ }^{3}$
}

\begin{abstract}
Severe hypercalcemia is a life-threatening condition. Hypercalcemia of malignancy can occur as a result of 4 different mechanisms, the most common mechanism being humoral hypercalcemia of malignancy (HHM). The second most common mechanism that majorly concerns our case is osteolytic hypercalcemia. In patients with osseous metastases, increased cytokine activity in the region of lytic osseous metastatic lesions greatly stimulate the activity of osteoclasts in the bone. When compensatory mechanisms are exceeded, the serum calcium level rises causing hypercalcemia. Symptoms and signs include nausea and vomiting, fatigue, depression, confusion, psychosis, abdominal pain, constipation, acute pancreatitis, peptic ulceration, polyuria/nocturia, hematuria, renal colic, renal failure, bone pain, hypertension, and arrhythmias. We present this case of severe hypercalcemia to emphasize the severity of disease associated with lytic skeletal metastasis with an emphasis on early management, diagnosis, and interventions to prevent early mortality.

Keywords: Hypercalcemia, Neoplasm, Vascular and metabolic disorders.

AMEI's Current Trends in Diagnosis \& Treatment (2020): 10.5005/jp-journals-10055-0094
\end{abstract}

\section{Case Description}

This is a 45-year-old female of Indian ethnicity who presented with the complain of intense pain in the lower back region associated with pain in the abdomen. For the past few months or so, she had noticed progressive decline in her ability to walk secondary to increasing muscle soreness and joint pains.

A physical examination revealed the following: stable vital signs; body temperature, $36^{\circ} \mathrm{C}$; respiratory rate, 20 breaths/minute; blood pressure, 122/68 $\mathrm{mm} \mathrm{Hg}$; heart rate, 140 beats/minute, regular rhythm; and no pathological murmurs. Per abdominal examination showed slight hepatomegaly with a span of $18 \mathrm{~cm}$ but smooth surface. As she was complaining of restlessness, her electrocardiogram was done, and sinus tachycardia with Qt interval shortening was noted with a QTc of $320 \mathrm{~m}$ second. Her serum calcium level on the date of admission was $18.42 \mathrm{mg} / \mathrm{dL}$ and plasma albumin level was $3.53 \mathrm{~g} / \mathrm{dL}$. Renal ultrasound did not reveal any stones or renal function compromise as ascertained by normal serum creatinine but showed suspicious lesions in both lobes of liver.

Patient was started on intravenous hydration with $0.9 \%$ normal saline of around $200 \mathrm{~mL} /$ hour, 1 dose of $4 \mathrm{mg}$ zoledronic acid, and injectable salmon calcitonin $4 \mathrm{lU} / \mathrm{kg}$ every 8 hours for 3 days. Zoledronic acid acts by inhibiting osteoclast-mediated bone resorption, hence preventing further release of calcium in bloodstream. Salmon calcitonin also inhibits osteoclast activity but primarily acts by inhibiting calcium absorption from intestine and renal tubules. Dexamethasone was also given. Patient started developing signs of encephalopathy on day 2 such that she started having altered talks and asterixis, so a session of hemodialysis was performed in view of metabolic encephalopathy, and the patient condition got stabilized. Third day serum calcium levels were brought down to $10.51 \mathrm{mg} / \mathrm{dL}$ successfully.

Concurrent to therapy, a contrast-enhanced CT of chest and abdomen was done on the patient delineating multiple subcentimetric lung nodules in bilateral lung fields and multiple heterogeneously enhancing lesions in both lobes of liver suggestive
${ }^{1}$ Department of Medical Oncology, Sri Guru Ram Das Rotary Cancer Hospital, Amritsar, Punjab, India

${ }^{2}$ Department of Medicine, Sri Guru Ram Das Institute of Medical Sciences and Research, Amritsar, Punjab, India

${ }^{3}$ Department of health, PHC lakhanpur Jammu and kashmir, india

Corresponding Author: Srishti Nayyar, Department of Medicine, Sri Guru Ram Das Institute of Medical Sciences and Research, Amritsar, Punjab, India, Phone: +91 9530854491, e-mail: srishtinayyar01@gmail. com

How to cite this article: Gupta R, Nayyar S, Mahajan S. Severe Lifethreatening Hypercalcemia in Skeletal Metastasis from Malignancy of Unknown Origin: A Rare Presentation. AMEl's Curr Trends Diagn Treat 2020;4(1):36-37.

Source of support: Nil

Conflict of interest: None

of neoplastic etiology. Destruction of body of sternum with cortical thinning was also seen. To ascertain a primary focus and extent of disease, an FDG-PET scan was performed on the patient, and while it confirmed extensive pulmonary, hepatic, skeletal, mediastinal, and abdominal lymph node metastasis, a primary could not be located. In view of skeletal metastasis, $120 \mathrm{mg}$ Denosumab, an inhibitor of osteoclast formation and function, was also administered to the patient to improve treatment response. Patient was discharged with calcium levels of $7.88 \mathrm{mg} / \mathrm{dL}$, serum creatinine levels of $1.41 \mathrm{mg} / \mathrm{dL}$, and stable condition. Palliative systemic chemotherapy based on nab-paclitaxel $280 \mathrm{mg}$ and carboplatin $450 \mathrm{mg}$ with monthly zoledronic acid is planned on follow-up with regular monitoring of serum electrolytes and renal function.

\section{Discussion}

Severe hypercalcemia is a life-threatening condition. Hypercalcemia is a serum calcium level above $2.62 \mathrm{mmol} / \mathrm{L}$ or $10.5 \mathrm{mg} / \mathrm{dL}$ 
(normal range $2.12-2.62 \mathrm{mmol} / \mathrm{L}$ or $8.5-10.5 \mathrm{mg} / \mathrm{dL}){ }^{1}$ It may be an asymptomatic laboratory finding. Symptoms usually become apparent with levels $>3.50 \mathrm{mmol} / \mathrm{L}$ or $14.03 \mathrm{mg} / \mathrm{dL}$. Primary hyperparathyroidism and malignancy account for $90 \%$ of cases. ${ }^{2}$ The historic incidence of hypercalcemia in the cancer population is noted to be as high as $20-30 \%$ and is associated with significant morbidity, including progressive cognitive dysfunction, dehydration, and acute renal failure in the patient. ${ }^{3}$ The most important component of evaluation is its identification, as hypercalcemia can very often be overlooked. The majority of calcium is bound to albumin. Free or ionized calcium is biologically active. Therefore, patients with low albumin levels and normal serum calcium levels actually may be having hypercalcemia. Therefore, it is necessary to use a corrected calcium (CRC) to accurately identify hypercalcemia (CRC = serum calcium $+0.8[4-$ serum albumin $] \mathrm{mg} / \mathrm{dL}){ }^{4}$

Goals of management of hypercalcemia of malignancy include measures to reduce the serum calcium levels rapidly, to restore the hydration status, and to maintain durable control. Aggressive hydration followed by administration of IV furosemide is helpful in causing rapid reduction in serum calcium levels. For rapid reduction in calcium levels, calcitonin should also be added. Calcitonin works by inhibiting renal tubular calcium reabsorption and blocking osteoclast activity. Its effect is rapid onset, but tachyphylaxis reduces the durability and limits its continued use beyond 2-3 days. ${ }^{5}$ Intravenous bisphosphonate therapy has become the mainstay of treatment to promote a more durable response and should be given as soon as possible in the absence of renal dysfunction. Bisphosphonates work by blocking osteoclast activity and decreasing bone turnover. However, bisphosphonates do not reach maximum effectiveness until days 2 through 4, highlighting the importance of proper hydration. ${ }^{6}$ Denosumab, a monoclonal antibody, is a RANK-L inhibitor and inhibits osteoclast formation, function, and survival and was helpful in our case owing to osteolytic pathology of hypercalcemia. Dialysis is reserved for patients who do not respond to conservative management or are developing neuropsychiatric symptomatology. Management of underlying pathology, such as in our case, with systemic palliative chemotherapy is of paramount importance to prevent recurrence.

\section{References}

1. Raftery AT, Lim E, Östör AJK. Hypercalcemia. Churchill's pocketbook of differential diagnosis. 2010;3e:506-507.

2. Cho KC. Electrolyte and acid base disorders. Curr Med Diagn Treat 2019;58e:919-946.

3. Stewart Andrew F. Clinical practice. Hypercalcemia associated with cancer. New Engl J Med 2005;352(4):373-379. DOI: 10.1056/ NEJMcp042806.

4. Malangone S, Campen CJ. Hypercalcemia of malignancy. J Adv Pract Oncol 2015;6(6):586-592.

5. Ralston SH, Gallacher SJ, Patel U, et al. Cancer-associated hypercalcemia: morbidity and mortality. Clinical experience in 126 treated patients. Ann Intern Med 1990;112(7):499-504. DOI: 10.7326/0003-4819-112-7-499.

6. Rodan GA, Fleisch HA. Bisphosphonates: mechanisms of action. J Clin Invest 1996;97(12):2692-2696. DOI: 10.1172/JCl118722. 